\title{
What are the participants' perspective and the system-based impact of a standardized, inter-professional morbidity/mortality- conferences in a children's hospital?
}

\author{
Martin Stocker ${ }^{1,2}$, Philipp Szavay ${ }^{3}$, Birgit Wernz ${ }^{4}$, Thomas J. Neuhaus ${ }^{2}$, Dirk Lehnick ${ }^{5}$, Sabine Zundel ${ }^{3}$ \\ ${ }^{1}$ Pediatric and Neonatal Intensive Care Unit, ${ }^{2}$ Department of Pediatrics, ${ }^{3}$ Department of Pediatric Surgery, ${ }^{4}$ Department of Nursing, Children's \\ Hospital Lucerne, Luzern, Switzerland; ${ }^{5}$ Biostatistics and Methodology, University of Lucerne, Luzern, Switzerland \\ Contributions: (I) Conception and design: M Stocker, S Zundel; (II) Administrative Support: P Szavay, B Wernz, TJ Neuhaus; (III) Provision of \\ study materials or patients: M Stocker, S Zundel; (IV) Collection and assembly of data: M Stocker, S Zundel, D Lehnick; (V) Data analysis and \\ interpretation: M Stocker, S Zundel, D Lehnick; (VI) Manuscript writing: All authors; (VII) Final approval of manuscript: All authors. \\ Correspondence to: Sabine Zundel. Department of Pediatric Surgery, Children's Hospital Lucerne, Spitalstrasse, CH-6000 Luzern 16, Switzerland. \\ Email: sabine.zundel@luks.ch.
}

Background: Morbidity and mortality conferences (MMC) are well established but little data exists on inter-professional aspects, system-based outcomes and characteristics in pediatric departments. Our study aim was to analyze the system-based impact and to assess participant's perspectives on standardized, interprofessional MMCs in a children's hospital.

Methods: In a prospective observational analysis the inter-professional MMCs held at a tertiary teaching children's hospital in Switzerland were analyzed for (I) resulting clinical consequences and (II) participants perception on format, usefulness and no-blame atmosphere.

Results: Eighteen MMC, discussing 29 cases were analyzed. Twenty-seven clinical errors/problems were identified and 17 clinical recommendations were developed: ten new or changed clinical guidelines, two new therapeutic alternatives, three new teaching activities, and two guidelines on specific diagnostics. Altogether, the 466 participants evaluated the conferences favorably. Little differences were seen in the evaluations of physicians of different disciplines or seniority but non-physicians scored all questions lower than physicians. Overall, three quarters of the participants felt that there was a no-blame culture during the conferences but results varied depending on the cases discussed.

Conclusions: An inter-professional MMC can have relevant impact on clinical practice and affect systembased changes. Inter-professional conferences are profitable for all participants but evaluated differently according to profession. A standardized format and the presence of a moderator are helpful, but not a guarantee for a no-blame culture. Highly emotional cases are a risk factor to relapse to "blame and shame". A time gap between the event and the MMC may have a beneficial effect.

Keywords: Inter-professional communication; inter-professional health care; learning from failure; morbidity and mortality conference (MMC); patient safety; psychological safety

Received: 13 January 2020; Accepted: 20 May 2020; Published: 25 July 2021.

doi: $10.21037 /$ tgh-20-42

View this article at: http://dx.doi.org/10.21037/tgh-20-42 


\section{Introduction}

The morbidity and mortality conference (MMC) has a longstanding tradition in health care. Originally, the aim of MMCs was to improve clinical management through casebased discussion and critical reflection among physicians $(1,2)$. The emphasis on errors and adverse events as a function of system performance led to a progressive shift of the original physician-centered MMC to a system-based quality improvement tool for patient safety (1-6). This fundamental change from a physician-centered educational tool to a health care management instrument opened the MMC for other professions $(4,5,7)$. The concept of an interprofessional MMC is in accordance with the demand for more inter-professional education as an essential strategy to improve inter-professional collaboration and patient safety (8-11).

For effective inter-professional collaboration and education, important profession-based differences of health care workers need to be considered $(12,13)$. In a children's hospital, goals for patient management strategies and on how to achieve them vary considerably between surgeons, pediatricians, and nurses. Differing perceptions and beliefs are known to be one of the main sources of conflict in health care $(14,15)$. Accordingly, goals and learning objectives of inter-professional activities may be rated differently among different disciplines and professions. Additionally, inter-professional communication often remains ineffective due to diverse communication styles and backgrounds, but effective communication is key for critical reflection, feedback and learning (15). Psychological safety is mandatory to enable health care workers to speak up, report and discuss errors. Perceived psychological safety is dependent on the individual as well as professional and organizational factors $(16,17)$. Acknowledgment of a noblame culture is dependent on perceived 'psychological safety' (16).

Currently, standards for inter-professional MMC are lacking and studies regarding participant's evaluation, especially in pediatric are scarce. All issues mentioned above need to be taken into account for an interprofessional MMC, otherwise they will hamper its success and effectiveness. Our study aim was (I) to analyze the system-based impact of a standardized, system-based, interprofessional MMC in a children's hospital and (II) to assess participant's perspectives on the format (standardized, system-based, inter-professional). We present the following article in accordance with the STROBE reporting checklist (available at http://dx.doi.org/10.21037/tgh-20-42).

\section{Methods}

\section{Setting}

The Children's Hospital of Lucerne is a tertiary teaching hospital in Switzerland. Regular continuing education for physicians and nurses includes lectures, journal clubs, small learning groups and in-situ simulation. A critical incident reporting system (CIRS) is implemented since 2000 and "speaking up" campaigns are regularly issued. Before July 2014, MMCs were held without a standardized format, interdisciplinary but mainly physician-based and on an irregular basis. In 2014, a new MMC format was developed and implemented by the newly formed MMC team consisting of two of the authors: SZ and MS. The SBAR format was applied, structuring the conferences in "situation - background - assessment - recommendations" as published and evaluated by Mitchell et al. $(18,19)$. Potential cases were defined as cases in need of cardiopulmonary resuscitation, cases with delayed diagnosis or suspected management errors and all fatal casualties except palliative cases. Beside cases within the safety I approach (learning from failure), enrolment of cases exemplary for the safety II approach (patients managed outstandingly well) were encouraged to enable learning from excellence (4,20-22). During the MMC, cases were presented according to SBAR by staff that had been directly involved $(18,19)$. Moderation was carried out by a member of the MMC team. It was the moderator's responsibility to guide the discussion, ensure a no blame atmosphere and assist in bringing out learning points and conclusions and write the minutes which were distributed to all hospital staff.

\section{Participants}

Physicians, nursing staff, midwifes, health-care co-workers, and medical students of diverse specialties working at the children's hospital or the perinatal department were involved. The MMCs were scheduled on a regular basis as continuing education activities and announcements of each MMC were done by email to the hospital staff.

\section{Analysis of the system-based impact}

Characteristics of each MMC and the system-based impact (error/problem classification and impact on clinical 
practice) were analyzed. Characteristics were grouped into: diagnostics, therapy, procedure-related, organization, communication, medication and documentation. Impact on clinical practice was defined as resulting new or changed guidelines, diagnostic recommendations, new therapies, new teaching activities, and changes in documentation.

\section{Analysis of the participant's perspective}

At the end of every MMC, a paper-based, anonymous questionnaire was distributed among the participants. The following demographic data was investigated: profession (physician, nursing staff, midwife, health-care co-worker, medical student or other), specialty (pediatrics, pediatric surgery or others); role (consultant or resident). Additionally, the following questions were asked: (I) Was this MMC helpful and will it influence your future practice? (II) Were important learning points defined and conclusions drawn? (III) Was the standardized presentation according to SBAR helpful? (IV) Was the presence of a moderator helpful? (V) Was there a no-blame culture? All answers were recorded within a 5-point Likert-scale. Answers within the Likert scale were tabulated between 0 and 2 (2: agree; 1.5: rather agree; 1 : neutral; 0.5 : rather disagree; and 0 : disagree) and mean values were calculated. Descriptive statistics was used for the analysis. In order to establish instrument validity, the questionnaire was tested on ten physicians who were interviewed about quality and ambiguity of questions.

\section{Results}

Between July 2014 and June 2017, we conducted 18 MMCs according to the new guideline. Twenty-nine patients were discussed within these 18 MMC. Twelve out of 29 cases were deceased patients (mortality conference: $41 \%$ ). In 26 out of 29 cases (90\%) the discussion was focused on learning from failure, in three cases $(10 \%)$ the discussion was focused on learning from excellence. The time between the event and the MMC was between one week and three years (median: three months). Throughout the 29 conferences, 27 separate errors were established as of critical importance for the case. These can be grouped into the following categories: diagnostics 8/27 (30\%), therapy 4/27 (15\%), procedure related $3 / 27(11 \%)$, organization 5/27 (18\%), communication 5/27 (18\%), medication $1 / 27$ (4\%), and documentation $1 / 27$ (4\%). Directly referring to these 27 errors, 17 clinical recommendations were developed and distributed: ten new or changed clinical guidelines, two new therapeutic alternatives, three new teaching activities, and two guidelines on specific diagnostics.

Four hundred and sixty-six health care workers participated at the $18 \mathrm{MMC}$ and completed the questionnaire ( 9 to 46 participants per MMC, the median being 24). These were: 164 (34\%) consultants, 144 (31\%) residents, $82(18 \%)$ medical students, and $79(17 \%)$ nurses, midwives and allied health care workers (non-physicians). There was a significant increase of the latter group from 19 participants (9\%) during the first 18 months to 60 participants $(22 \%)$ during the second 18 months $(\mathrm{P}<0.001)$.

In general the MMCs were evaluated favorably with participants rating "agree $=2$ " and "rather agree $=1.5$ " most often (Figure 1A). The highest score (1.59) was achieved for perceived "no-blame culture" (psychological safety). Slightly lower scores were achieved regarding presentation format (1.39) and presence of a moderator (1.56). The lowest scores were given regarding the content of the MMC: helpful for future practice (1.21) and important conclusions shown (1.29) (Figure 1A). Analyzing this data by profession, role and discipline, no differences were found between pediatrics and pediatric surgery and roles (consultant/resident/student) but distinctively for profession. Non-physicians scored all questions except the question regarding the moderator lower than physicians. Pediatricians scored overall highest compared to all other groups (Figure 1A).

Comparing the 18 conferences individually, we found conferences with overall good ratings $(>1.5)$, highest for conference number 9 , and conferences with overall low ratings $(<1)$ : conferences number 2, 5, and 7 (Figure 1B). Some conferences showed low ratings in especially one area as conference number 13 for no-blame culture. The difference between the best and the worst rated MMC was highest for the question regarding no blame culture (difference of 1.72 between MMC 4 and 13), lower regarding the content of the MMC (difference of 1.35 for the question regarding helpfulness and 1.36 for the question regarding conclusions), and lowest regarding the communication style (difference of 1.06 for the format and 0.96 for the presence of a moderator). Three quarters of the participants felt that there was a no-blame culture during the conferences. Notably, the lower ratings came from nonphysicians. Additionally, the question about the no-blame culture achieved the overall highest score, but the highest difference between mean scores (best $=1.95$ and worst $=0.24$ ). As shown in figure $1 \mathrm{~b}$, there were three MMCs with very low scores regarding a no-blame culture (conferences 2, 7 , and 13), all other conferences scored $>1.5$. 


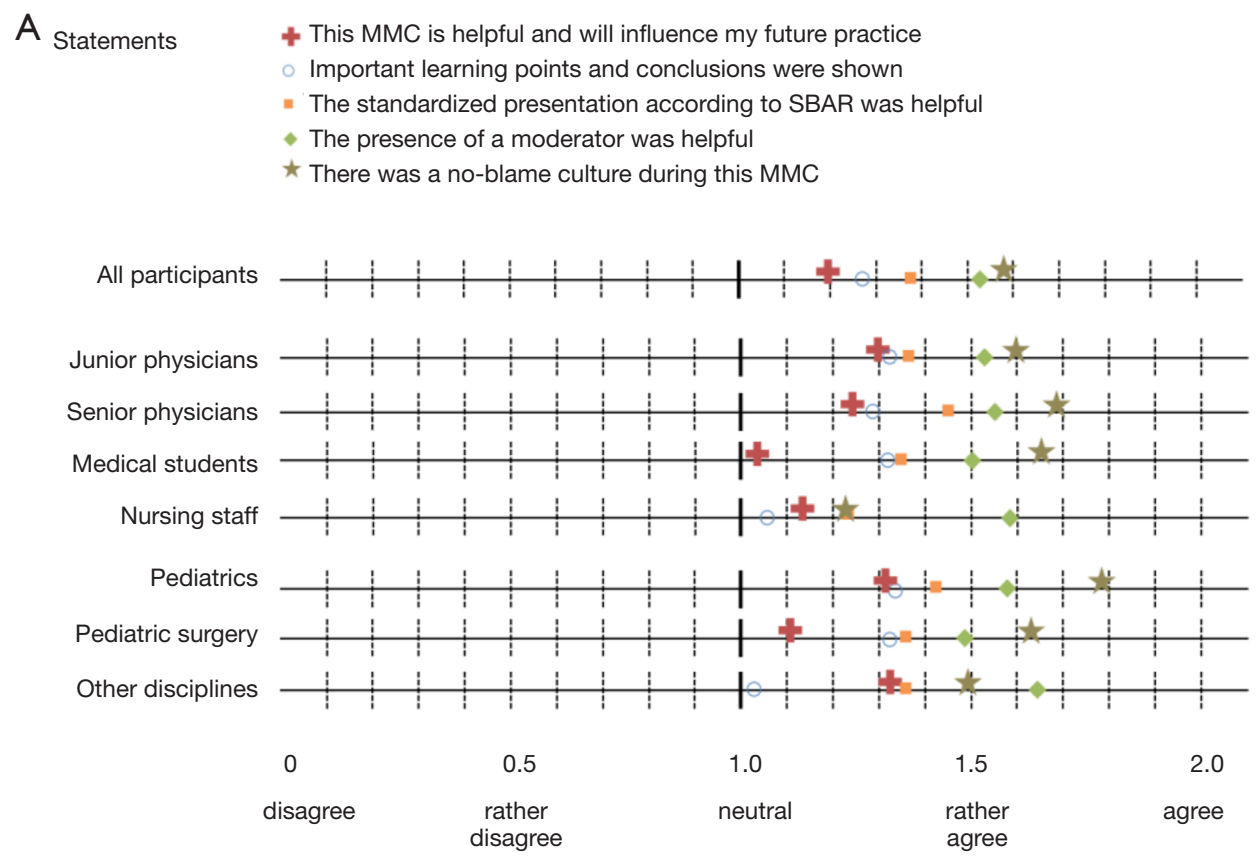

B

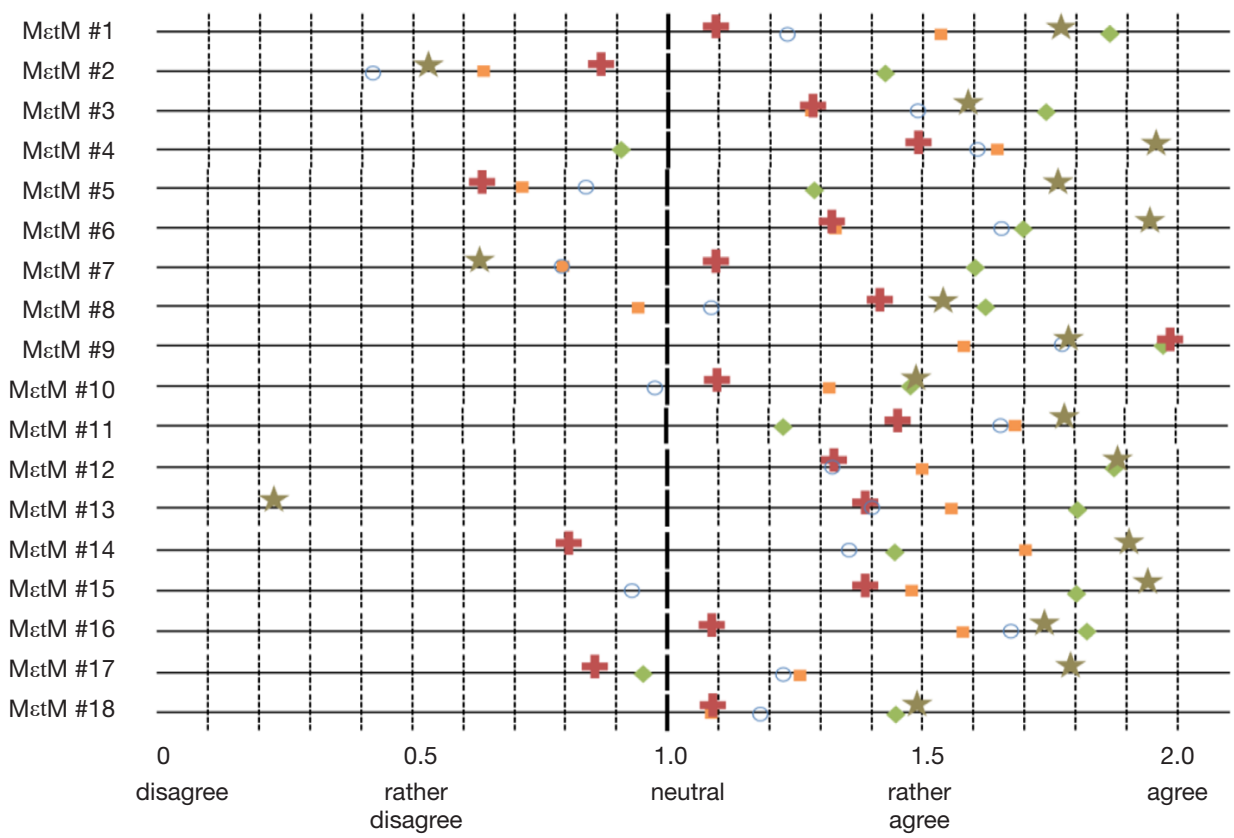

Figure 1 Questionnaire results by participant groups and conference: (A) evaluation of all conferences by participant groups; (B) evaluation of single conferences by participants overall.

\section{Discussion}

The analyses of our inter-professional MMCs showed a relevant impact on clinical practice with the discovery of 27 errors resulting in the implementation of 17 recommendations. Furthermore, the results confirm that an inter-professional activity is perceived differently by participants depending on their profession. MMCs are a potential educational tools $(3,5,18,23-25)$, but learning from mistakes is challenging. "If the effort is successful, it can 
serve as a model. If unsuccessful, it is at best unproductive and at worst unpleasant" (26). There are few studies published on pediatric MMC, most focus on accordance of clinical diagnoses and post-mortem findings, few concentrate on methodology, participants' perspective and clinical impact $(5,7,23,27-30)$.

When analyzing the generally very good evaluation in detail, the slightly lower scores and wide distribution of scores regarding the questions about the content is notable. This is directly linked to the inter-professionalism of the conferences. Lower ratings on "will this influence my future practice" by non-pediatric physicians is most likely due to the assumption that they will not encounter cases like the discussed ones regularly. The lower ratings by nonphysicians may be due to a different perspective on the care they provide. This theory is in line with the literature: perspective and personality traits of physicians and nurses have been described as drivers and potential sources of conflicts (31). To make the conferences more relevant to the practice of all professions present, the moderators should be instructed to take the various interests into account.

Some studies show a divergent perception on MMCs when comparing opinions of junior and senior physicians $(32,33)$. In our study, both residents and consultants assessed the MMCs very similarly. The reason for this divergence to the literature is unclear; possible reasons might be the influence of the study population, the zeitgeist and local characteristics including the Swiss educational system. The previous studies have not been performed in a pediatric setting, or in Switzerland, and they were done more than a decade ago.

When comparing the evaluation results for the individual MMCs, the ratings for content show a great variability. We believe this to be directly depended on the cases dealt with. In retrospect, some of the cases discussed were very specific and the relevance for the whole children's hospital might have been overrated prior to the conference. On the other hand, the high ratings on some of our MMCs show that it is possible to conduct inter-professional MMCs with a content relevant for all participants.

The presence of a moderator was overall agreed to be important and had the highest score by members of the nursing staff and "physicians of other specialties". The variability between individual conferences was small which emphasizes that participants appreciated the moderator independent of individual conference. This is in line with the literature showing that a standard format and a moderator are helpful $(34,35)$. Non-physicians are hesitant to speak up when physicians are present $(36,37)$. Physicians working mainly outside the children's hospital may have similar reservations because they are ignorant of the local hidden curriculum. Fear to attract negative attention or cause negative emotions in others might explain their reservations. In contrast, physicians working together on a daily basis are unlikely to have scruple to voice their opinion in direct confrontation. Co-workers' opinion on each other is already formed, multi-layered from countless encounters and will not be influenced by a single comment in a MMC (38). The moderator should balance the tendency for outspoken individuals to dominate the rhetoric and encourage cautious participants. However, the role of the moderator regarding perceived psychological safety is difficult to assess. Some conferences had the same high rating for presence of the moderator and no-blame culture, whereas others achieved a high rating for one and a low rating for the other question. MMC 4 may serve as an example with a high rated psychological safety and a low rating for the moderator, whereas MMC number 13 achieved a very high rating for the moderator and a very low value for psychological safety. This might be due to the fact that a valued moderator is no guarantee for a no-blame culture. On the other hand, this finding might be a tool related mistake. The suggested answers on the questionnaire might have not been able to capture participants' opinions fully. Better data would have been generated by using open ended questions.

When analyzing the three conferences with low ratings on the no-blame question, we found, that during these conferences, highly emotional discussions between physicians of different disciplines took place. Perspectives of different disciplines are known to be divergent and there is some literature on personality characteristics of different specialties i.e. surgeons, non-surgeons and nurses, but if this is causal remains speculation. Detailed interviews on the reason for the perceived failure of non-blame would have generated more multi-layered data. Additionally, we found that the time interval between the event and the conference was comparably short for the conferences with these low rankings (between two and five weeks). We hypothesize that these might have been scheduled too early to facilitate a non-emotional focused discussion. This is supported by the findings from one other conference: conference 11 was expected to be difficult and emotional since it dealt with a sudden and unexpected death of a newborn. The conference was held ten months after the event and it achieved very high ratings for content and no-blame culture. There is 
no data regarding the best time for MMCs. Debriefings after resuscitations and adverse events are recommended to be done shortly after the event since they deal with participants' emotions aiming to avoid fear and feelings of guilt. The aim of a MMC conference is different-focusing on generalization and learning points. To allow work on these objectives, a different timeline seems to be preferable.

\section{Conclusions}

A system-based, inter-professional MMC can have a relevant impact on clinical practice and effect system-based changes.

The ideal time for MMC discussions seems to be between three and nine months to capitalize on recollection of events but avoid some of the emotional issues that can derail the purpose of the meeting. A moderator with no direct relationship to the events being discussed allows for objective questioning and encourages input from multiple participants. Using a standardized format ensures a consistent delivery of data and facilitates important discussion.

\section{Acknowledgments}

Funding: None.

\section{Footnote}

Provenance and Peer Review: This article was commissioned by the Guest Editors (Eduardo Perez, Samir Pandya, and Matthew S. Clifton) for the Focused Series "Current Topics in Pediatric General Surgery" published in Translational Gastroenterology and Hepatology. The article has undergone external peer review.

Reporting Checklist: The authors have completed the STROBE reporting checklist. Available at http://dx.doi. org/10.21037/tgh-20-42.

Data Sharing Statement: Available at http://dx.doi. org/10.21037/tgh-20-42.

Conflicts of Interest: All authors have completed the ICMJE uniform disclosure form (available at http://dx. doi. org/10. 21037/tgh-20-42). The Series "Current Topics in Pediatric General Surgery" was commissioned by the editorial office without any funding or sponsorship. The authors have no other conflicts of interest to declare.

Ethical Statement: The authors are accountable for all aspects of the work in ensuring that questions related to the accuracy or integrity of any part of the work are appropriately investigated and resolved. The study was conducted in accordance with the Declaration of Helsinki (as revised in 2013). According to the Swiss law (Humanforschungsgesetz) questionnaires asking health care workers do not need to be approved by a Swiss Ethical Board. This is in accordance to the published guidelines of the British Educational Research Association. Written informed consent to analyze and publish the data was obtained from all participants. A copy of the written consent is available for review.

Open Access Statement: This is an Open Access article distributed in accordance with the Creative Commons Attribution-NonCommercial-NoDerivs 4.0 International License (CC BY-NC-ND 4.0), which permits the noncommercial replication and distribution of the article with the strict proviso that no changes or edits are made and the original work is properly cited (including links to both the formal publication through the relevant DOI and the license). See: https://creativecommons.org/licenses/by-nc-nd/4.0/.

\section{References}

1. Morbiditäts-und Mortalitätskonferenzen in der Schweiz.. Accessed 27 Oct 2017. Available online: https://saez.ch/de/ article/doi/bms.2017.06056/.

2. Gregor A, Taylor D. Morbidity and mortality conference: its purpose reclaimed and grounded in theory. Teach Learn Med 2016;28:439-47.

3. Bechtold ML, Scott S, Dellsperger KC, et al. Educational quality improvement report: outcomes from a revised morbidity and mortality format that emphasised patient safety. Postgrad Med J 2008;84:211-6.

4. Fraser J. The morbidity and mortality meeting: time for a different approach? Arch Dis Child 2016;101:4-8.

5. Frey B, Doell C, Klauwer D, et al. The morbidity and mortality conference in pediatric intensive care as a means for improving patient safety. Pediatr Crit Care Med 2016;17:67-72.

6. Gonzalo JD, Yang J, Huang G. Systems-based content in medical morbidity and mortality conferences. A decade of change. J Grad Med Educ 2012;4:438-44.

7. Cifra CL, Jones KL, Ascenzi JA, et al. Diagnostic errors 
in a PICU: insights from the morbidity and mortality conference. Pediatr Crit Care Med 2015;16:468-76.

8. Jeffs L, Abramovich IA, Hayes C, et al. Implementing an interprofessional patient safety learning initiative: insights from participants, project leads and steering committee members. BMJ Qual Saf 2013;22:923-30.

9. Leape L, Berwick D, Clancy C, et al. Transforming healthcare: a safety imperative. Qual Saf Health Care 2009;18:424-8.

10. Palaganas JC, Epps C, Raemer DB. A history of simulation-enhanced interprofessional education. J Interprof Care 2014;28:110-5.

11. Stocker M, Pilgrim SB, Burmester M, et al. Interprofessional team management in pediatric critical care: some challenges and possible solutions. J Multidiscip Healthc 2016;9:47.

12. House S, Havens D. Nurses' and physicians' perceptions of nurse-physician collaboration: a systematic review. J Nurs Adm 2017;47:165-71.

13. Reeves S, Lewin S, Espin S, et al. Interprofessional teamwork for health and social care. John Wiley \& Sons; 2011.

14. Kim S, Bochatay N, Relyea-Chew A, et al. Individual, interpersonal, and organisational factors of healthcare conflict: a scoping review. J Interprof Care 2017;31:282-90.

15. Tan TC, Zhou H, Kelly M. Nurse-physician communication-An integrated review. J Clin Nurs 2017;26:3974-89.

16. Edmondson AC. Teaming: How organizations learn, innovate, and compete in the knowledge economy. John Wiley \& Sons; 2012.

17. Okuyama A, Wagner C, Bijnen B. Speaking up for patient safety by hospital-based health care professionals: a literature review. BMC Health Serv Res 2014;14:61.

18. Mitchell EL, Lee DY, Arora S, et al. Improving the quality of the surgical morbidity and mortality conference: a prospective intervention study. Acad Med 2013;88:824-30.

19. Mitchell EL, Lee DY, Arora S, et al. SBAR M\&M: a feasible, reliable, and valid tool to assess the quality of, surgical morbidity and mortality conference presentations. The Am J Surg 2012;203:26-31.

20. Kelly N, Blake S, Plunkett A. Learning from excellence in healthcare: a new approach to incident reporting. Arch Dis Child 2016;101:788-91.

21. Patterson M, Deutsch ES. Safety-I, Safety-II and resilience engineering. Curr Probl Pediatr Adolesc Health Care 2015;45:382-9.
22. Staender S. Safety-II and resilience: the way ahead in patient safety in anaesthesiology. Curr Opin Anaesthesiol 2015;28:735-9.

23. Cifra CL, Bembea MM, Fackler JC, et al. Transforming the morbidity and mortality conference to promote safety and quality in a pediatric intensive care unit. Pediatr Crit Care Med 2016;17:58.

24. Giesbrecht V, Au S. Morbidity and mortality conferences: a narrative review of strategies to prioritize quality improvement. Jt Comm J Qual Patient Saf 2016;42:516-27.

25. Rabizadeh S, Gower WA, Payton K, et al. Restructuring the morbidity and mortality conference in a department of pediatrics to serve as a vehicle for system changes. Clin Pediatr 2012;51:1079-86.

26. Orlander JD, Barber TW, Fincke BG. The morbidity and mortality conference: the delicate nature of learning from error. Acad Med 2002;77:1001-6.

27. Cifra CL, Bembea MM, Fackler JC, et al. The morbidity and mortality conference in PICUs in the United States: a national survey. Crit Care Med 2014;42:2252-7.

28. Cifra CL, Jones KL, Ascenzi J, et al. The morbidity and mortality conference as an adverse event surveillance tool in a paediatric intensive care unit. BMJ Qual Saf 2014;23:930-8.

29. Friedman JN, Pinard MS, Laxer RM. The morbidity and mortality conference in university-affiliated pediatric departments in Canada. J Pediatr 2005;146:1-2.

30. Widmann R, Caduff R, Giudici L, et al. Value of postmortem studies in deceased neonatal and pediatric intensive care unit patients. Virchows Archiv 2017;470:217-23

31. Eley DS, Eley RM. Personality traits of Australian nurses and doctors: Challenging stereotypes? Int J Nurs Pract 2011;17:380-7.

32. Harbison SP, Regehr G. Faculty and resident opinions regarding the role of morbidity and mortality conference. The American journal of surgery 1999;177:136-9.

33. Murayama KM, Derossis AM, DaRosa DA, et al. A critical evaluation of the morbidity and mortality conference. Am J Surg 2002;183:246-50.

34. de Vos MS, Marang-van de Mheen PJ, Smith AD, et al. Toward best practices for surgical morbidity and mortality conferences: a mixed methods study. J Surg Educ 2018;75:33-42.

35. Higginson J, Walters R, Fulop N. Mortality and morbidity meetings: an untapped resource for improving the governance of patient safety? BMJ Qual 
Saf 2012;21:576-85.

36. Alingh CW, van Wijngaarden JD, van de Voorde K, et al. Speaking up about patient safety concerns: the influence of safety management approaches and climate on nurses' willingness to speak up. BMJ Qual Saf 2019;28:39-48.

37. Morrow KJ, Gustavson AM, Jones J. Speaking up

doi: $10.21037 / \operatorname{tgh}-20-42$

Cite this article as: Stocker M, Szavay P, Wernz B, Neuhaus TJ, Lehnick D, Zundel S. What are the participants' perspective and the system-based impact of a standardized, interprofessional morbidity/mortality-conferences in a children's hospital? Transl Gastroenterol Hepatol 2021;6:48. behaviours (safety voices) of healthcare workers: a metasynthesis of qualitative research studies. Int J Nurs Stud 2016;64:42-51.

38. Bennett N, Lockyer J, Mann K, et al. Hidden curriculum in continuing medical education. J Contin Educ Health Prof 2004;24:145-52. 